\title{
MCT8 expression in human fetal cerebral cortex is reduced in severe intrauterine growth restriction
}

\author{
Shiao Y Chan', Laura A Hancox', Azucena Martín-Santos', Laurence S Loubière', \\ Merlin N M Walter', Ana-Maria González', Phillip M Cox², Ann Logan', \\ Christopher J McCabe', Jayne A Franklyn' and Mark D Kilby ${ }^{1,3}$
}

${ }^{1}$ School of Clinical and Experimental Medicine, College of Medical and Dental Sciences, University of Birmingham, Edgbaston, Birmingham B15 2TT, UK

${ }^{2}$ Department of Pathology and ${ }^{3}$ Fetal Medicine Centre, Birmingham Women's NHS Foundation Trust, Edgbaston, Birmingham B15 2TG, UK
Correspondence should be addressed to S Y Chan

Email

s.chan@bham.ac.uk

\begin{abstract}
The importance of the thyroid hormone (TH) transporter, monocarboxylate transporter 8 (MCT8), to human neurodevelopment is highlighted by findings of severe global neurological impairment in subjects with MCT8 (SLC16A2) mutations. Intrauterine growth restriction (IUGR), usually due to uteroplacental failure, is associated with milder neurodevelopmental deficits, which have been partly attributed to dysregulated TH action in utero secondary to reduced circulating fetal $\mathrm{TH}$ concentrations and decreased cerebral thyroid hormone receptor expression. We postulate that altered MCT8 expression is implicated in this pathophysiology; therefore, in this study, we sought to quantify changes in cortical MCT8 expression with IUGR. First, MCT8 immunohistochemistry was performed on occipital and parietal cerebral cortex sections obtained from appropriately grown for gestational age (AGA) human fetuses between 19 weeks of gestation and term. Secondly, MCT8 immunostaining in the occipital cortex of stillborn IUGR human fetuses at 24-28 weeks of gestation was objectively compared with that in the occipital cortex of gestationally matched AGA fetuses. Fetuses demonstrated widespread MCT8 expression in neurons within the cortical plate and subplate, in the ventricular and subventricular zones, in the epithelium of the choroid plexus and ependyma, and in microvessel wall. When complicated by IUGR, fetuses showed a significant fivefold reduction in the percentage area of cortical plate immunostained for MCT8 compared with AGA fetuses $(P<0.05)$, but there was no significant difference in the proportion of subplate microvessels immunostained. Cortical MCT8 expression was negatively correlated with the severity of IUGR indicated by the brain:liver weight ratios $\left(r^{2}=0.28 ; P<0.05\right)$ at post-mortem. Our results support the hypothesis that a reduction in MCT8 expression in the IUGR fetal brain could further compromise TH-dependent brain development.
\end{abstract}

\author{
Key Words \\ - мСт8 \\ - human fetus \\ - CNS \\ - intrauterine growth \\ restriction (IUGR)
}

Journal of Endocrinology (2014) 220, 85-95

\section{Introduction}

Intrauterine growth restriction (IUGR) describes the failure of a fetus to attain its genetically determined growth potential, with the most common underlying etiology being uteroplacental failure associated with abnormal placental development. IUGR is often characterized by continued head and brain growth at the expense of other 
less vital organs resulting in an elevated brain:liver weight ratio postnatally (Cox \& Marton 2009). IUGR complicates $5-10 \%$ of pregnancies and is associated with increased perinatal mortality (Kady \& Gardosi 2004). Survivors demonstrate an increased prevalence of cognitive impairment compared with babies born appropriately grown for gestational age (AGA). They have lower school achievements and intelligence quotient (IQ) scores (Leitner et al. 2007), and 5\% show neurodevelopmental delay at the age of 9-10 years (Kok et al. 1998). Significantly reduced circulating concentrations of free thyroxine $\left(\mathrm{T}_{4}\right)$ and tri-iodothyronine $\left(\mathrm{T}_{3}\right)$ (Kilby et al. 1998) and decreased expression of cerebral thyroid hormone receptor (TR) expression (Kilby et al. 2000) in growth-restricted human fetuses are postulated to contribute to this neurodevelopmental morbidity. Examination of growth-restricted fetal guinea pigs has shown a compensatory increase in brain deiodinase type 2 (DIO2) expression, which could increase local concentrations of the active thyroid hormone (TH) ligand, $T_{3}$, from $T_{4}$ conversion (Chan et al. 2005). In clinical practice, once IUGR is diagnosed antenatally, timely delivery aimed at avoiding in utero demise while prolonging gestation as far as possible for fetal maturity is the mainstay of management. Currently, there are no in utero therapies to reduce the risk of neurocognitive impairment in IUGR. An increased understanding of how TH-responsive neurodevelopment is altered in IUGR may lead to the development of novel therapies to improve long-term outcome.

Monocarboxylate transporter 8 (MCT8) is a highly specific plasma membrane TH transporter (Friesema et al. 2003). Its importance to human CNS development has been highlighted by discoveries of different mutations within the MCT8 gene (SLC16A2) in subjects with a variety of X-linked mental retardation syndromes, characterized by severe psychomotor and cognitive impairment and accompanied by elevated serum free $T_{3}$ concentrations but normal or low free $\mathrm{T}_{4}$ concentrations (Dumitrescu et al. 2004, Friesema et al. 2004, Schwartz et al. 2005).

In mice, MCT8 facilitates the entry of TH into the brain parenchyma across the blood brain-barrier (Ceballos et al. 2009) and, at a cellular level, the entry of TH into neurons (Trajkovic et al. 2007), where MCT8 is responsible for $75 \%$ of $\mathrm{T}_{3}$ uptake (Wirth et al. 2009). In rodents, $\mathrm{TH}$ affects cell proliferation and differentiation of neuroblastoma cells (Garcia-Silva et al. 2002) and oligodendrocytes (Jones et al. 2003), neuronal migration (Lavado-Autric et al. 2003, Auso et al. 2004), synaptogenesis (Gilbert \& Paczkowski 2003), and cerebellar Purkinje cell dendritic outgrowth (Heuer \& Mason 2003). $\mathrm{T}_{3}$ has a proproliferative effect in human neuronal precursor cells, NT2, but MCT8, independently of $T_{3}$, could repress NT2 proliferation (James et al. 2009), suggesting another role for MCT8 apart from TH transport. However, the lack of neurological defects in Mct8-knockout mice (Wirth et al. 2009) emphasizes the need for studies in humans. From 7 weeks of gestation, the human fetal cerebral cortex is potentially $\mathrm{TH}$ responsive, expressing a range of $\mathrm{TH}$ transporters including MCT8 (Chan et al. 2011) and all the major TR isoforms (nuclear transcription factors that bind to $\mathrm{T}_{3}$ to regulate gene transcription), and demonstrates pre-receptor regulation by $\mathrm{DIO} 2$ and deiodinase type 3 (DIO3) (which inactivates $\mathrm{T}_{4}$ and $\mathrm{T}_{3}$ ) (Chan et al. 2002). Fetal neurons are believed to be the main target for TH action in the brain.

We hypothesize that human fetal cortical MCT8 expression is reduced with severe IUGR, which could further compromise neurodevelopment. In this study, we first carried out localization studies for MCT8 expression in the human fetal cerebral cortex from mid-gestation onwards. We then compared cortical МСТ8 expression in severe IUGR human fetuses with that in AGA human fetuses that were stillborn.

\section{Subjects and methods}

\section{Brain samples}

This study was approved by the South Birmingham Research Ethics Committee. Written consent for blocks and slides to be used in research and teaching was obtained in all cases. Cases were identified retrospectively from reports of all post-mortems conducted at the Birmingham Women's Hospital over a 3-year period. Only a minority of cases fulfilled our strict inclusion criteria: normal karyotype, no histopathological evidence of intrauterine infection, and limited or no maceration (indicating very short death-to-delivery intervals). Gestational ages were determined by first-trimester ultrasound scan for crown-rump length. Sections of formalin-fixed paraffin-embedded (FFPE) samples were then obtained from the hospital archive of histopathology blocks.

First, sections of the fetal cerebral cortex (occipital and parietal) obtained during the second (19-20 weeks; $n=3$ ) and third (26-37 weeks; $n=3$ ) trimesters from AGA fetuses with unexplained intrauterine deaths were examined. Sections of normal adult occipital cortex (one female aged 55 years and one male aged 37 years) sampled at postmortem and donated to the London Neurodegenerative

Published by Bioscientifica Ltd

Downloaded from Bioscientifica,com at $04 / 26 / 2023$ 09:40:40AM via free access 
Diseases Brain Bank (King's College London, Institute of Psychiatry) were obtained for comparison.

Secondly, sections of the occipital cerebral cortex obtained from stillborn human fetuses between 24 and 28 weeks of gestation were obtained and categorized as either IUGR $(n=7)$ or AGA $(n=5)$ (Table 1). IUGR was defined as having i) a birth weight below the third percentile for gestation, based on customized growth charts, which account for maternal weight, height, parity, ethnicity, gestation, and fetal sex (Gardosi et al. 1992) and ii) a brain:liver weight ratio $>4$ (Cox \& Marton 2009). Although we had not prospectively documented the presence of fetal growth restriction prenatally before death, the post-mortem features were highly suggestive of this pathology. IUGR is likely to be significant, as the phenotype was associated with fetal demise.

\section{Immunohistochemistry}

FFPE sections $(5 \mu \mathrm{m})$ of cortical samples were immunostained for MCT8 using an avidin-biotin peroxidase technique (Vectastain Elite; all reagents from Vector Laboratories (Peterborough, UK) unless otherwise stated) as per the kit instructions as described previously (Chan et al. 2011). Briefly, after dewaxing and serial rehydration, the sections were incubated in $10 \mathrm{mM}$ sodium citrate buffer (pH 6.0) in a $95^{\circ} \mathrm{C}$ water bath for $10 \mathrm{~min}$. After washing in $50 \mathrm{mM}$ Tris/0.15 M saline (pH 7.5; TBS), the sections were blocked with $10 \%$ normal goat serum (Sigma-Aldrich) in diluting buffer (TBS, $0.3 \%$ Tween 20 , and $2 \%$ BSA) for $20 \mathrm{~min}$. Then, consecutive sections were incubated overnight at $4{ }^{\circ} \mathrm{C}$ with rabbit anti-MCT8 (4790) (SigmaGenosys Ltd., Haverhill, UK; Vasilopoulou et al. 2010, Chan et al. 2011) at a concentration of $1 \mu \mathrm{g} / \mathrm{ml}$, anti-glial fibrillary acidic protein (GFAP, glia marker; Dako M0761 at 1:120 (Carpinteria, CA, USA)), or anti-CD68 (microglia marker; Dako M0876 at 1:100). The sections were incubated with biotinylated goat anti-rabbit secondary antibody at 1:200 for 30 min followed by incubation in $5 \%$ HRP for $5 \mathrm{~min}$ and then in the avidin-biotinperoxidase complex for $30 \mathrm{~min}$. Immunoreactivity was visualized with $3,3^{\prime}$-diaminobenzidine ( $\left.15 \mathrm{~min}\right)$. All the steps were separated by TBS-Tween washes. Sections used for localization studies were lightly counterstained with Mayer's hematoxylin and mounted in Vectamount. Slides for comparisons of IUGR fetuses with AGA fetuses were mounted with aqueous Vectashield H1000 without counterstaining. The sections were examined using bright-field microscopy using a Zeiss microscope, and images were captured using the AxioVision Software (Oberkochen,
Germany). The specificity of this MCT8 antiserum (4790) has been determined previously (Vasilopoulou et al. 2010, Chan et al. 2011) and confirmed in the studies by preincubating the primary antibody with blocking peptide $(25 \mu \mathrm{g} / \mathrm{ml})$ before application to adjacent sections. Negative control immunostaining was also performed for each tissue sample by omitting the primary antibody.

\section{Quantification of MCT8 immunostaining}

Comparisons of IUGR fetuses with AGA fetuses focused on the occipital cerebral cortex, which is involved in visual perception and interpretation. The in utero development of this structure is thought to be TH responsive (Zoeller \& Rovet 2004) and affected in IUGR (Dowdeswell et al. 1995). We quantified i) the percentage area of cortical plate immunostained for MCT8 and ii) the proportion of microvessels stained for MCT8 in the subplate, with the researcher blinded to the experimental grouping.

MCT8 staining in the cortical plate For each fetus, five images ( $20 \times$ magnification) from the MCT8immunostained section and five corresponding images from the adjacent section processed with the omission of the primary antibody as a negative control were analyzed. An objective measure of the area containing brown pixels corresponding to immunoreactive staining for MCT8 was quantified using the software ImageJ (U. S. National Institutes of Health, Bethesda, MD, USA) as described previously (Ferreira \& Rasband 2012). Briefly, bright-field images were converted to grayscale 'RGB stack', and the green channel image was used for analysis. A grayscale cutoff point derived from the corresponding negative controls was set as the threshold signifying positive staining and the same threshold applied to the immunostained sections for each fetus. The total area of tissue stained above the threshold was quantified and expressed as a proportion of the total tissue area examined. The area fraction of background noise, as determined by applying the same threshold to the corresponding negative control, was subtracted from the area fraction of tissue stained to give the true proportion of area of tissue staining positively for MCT8. Since the area of staining could also be affected by cell density, the number of cell bodies (nuclei) within a $250 \times 250$ pixel field in each quadrant of every image analyzed was counted and averaged to determine the relative cellularity, which was used to correct the area stained for MCT8. The corrected percentage of area stained for each fetus was then expressed relative to the mean of the AGA group, which was assigned an arbitrary value of 1 .

Published by Bioscientifica Ltd 


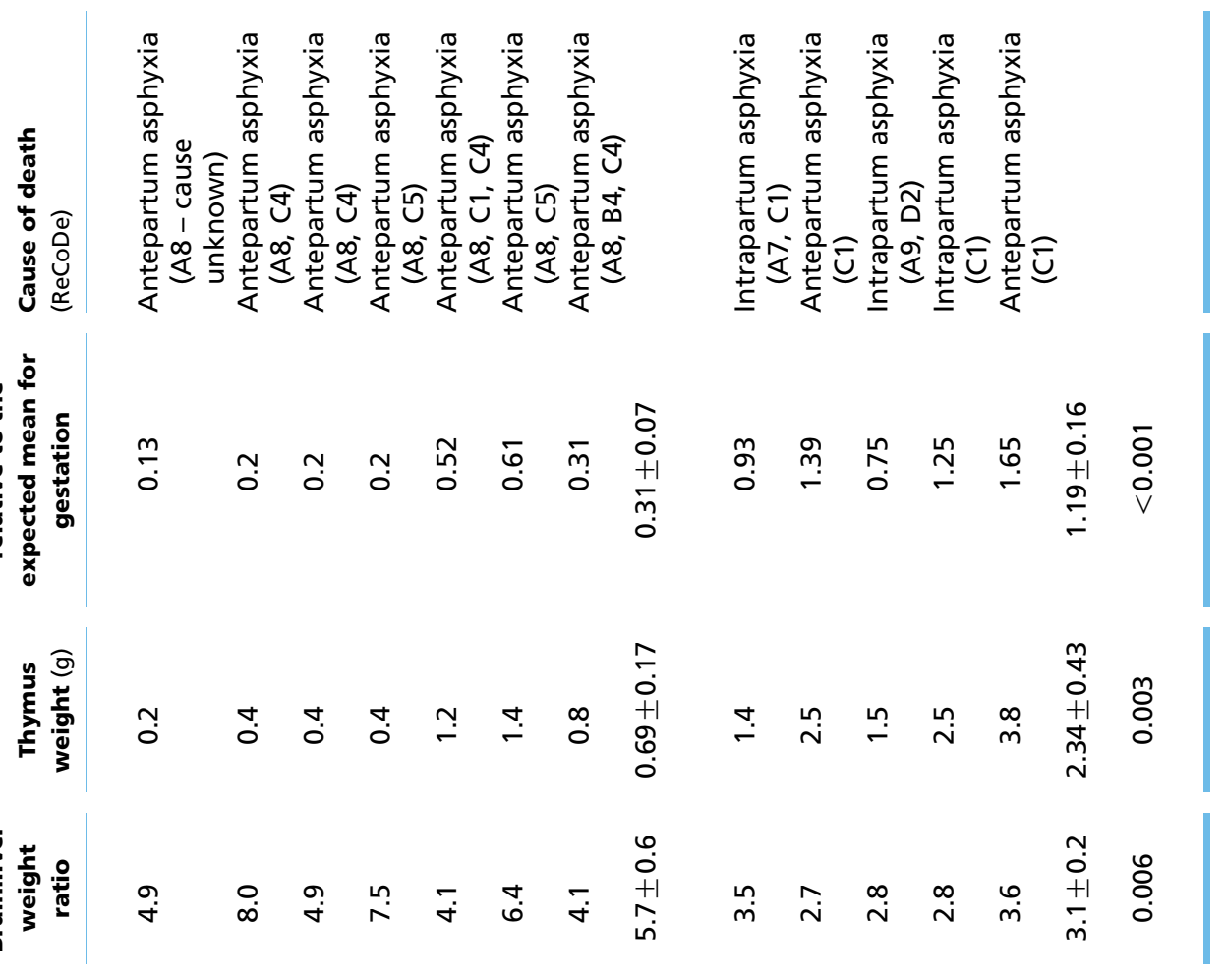

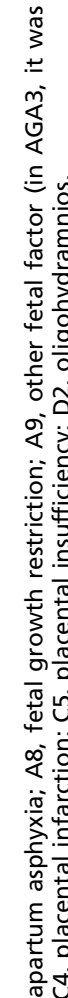

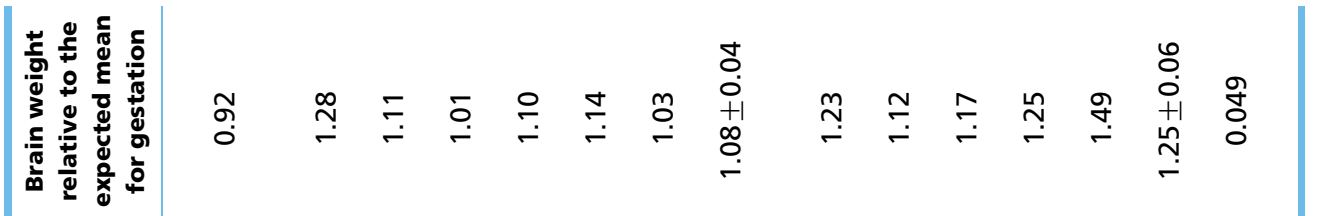

|

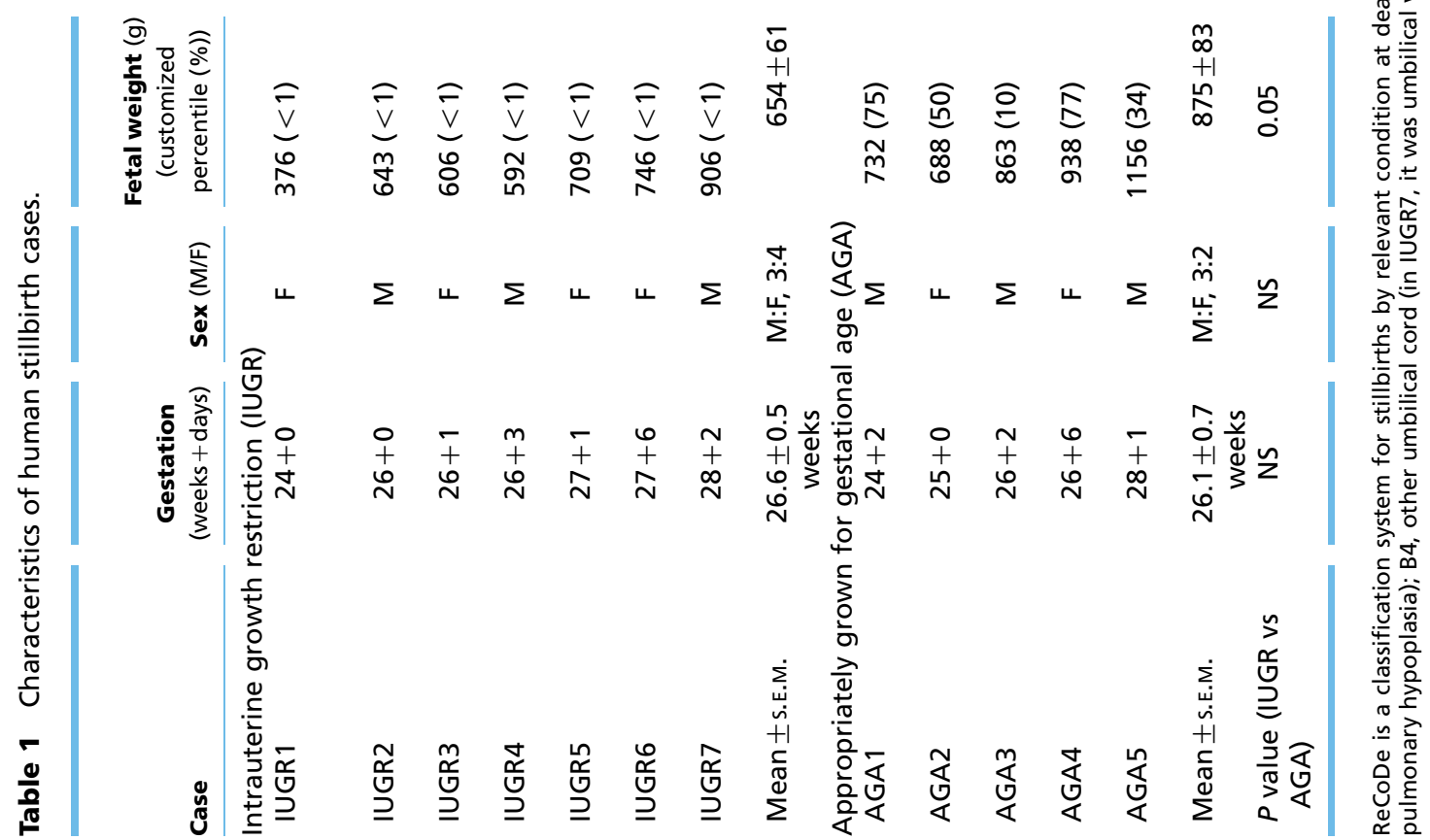


MCT8 staining in microvessels MCT8 immunoreactivity in microvessels was assessed in the subplate zone, a layer deep to the cortical plate with a lower density of cells, where it was easily possible to identify all the microvessels in bright field based on morphology at $40 \times$ magnification. For each fetus, 20 non-overlapping images of the subplate were taken. The number of immunostained microvessels was counted and calculated as a percentage of all the microvessels present. An average of $40.4 \pm 1.9$ microvessels was counted per fetus. Non-specific staining of intravascular erythrocytes was disregarded. The percentage of microvessels stained was then expressed relative to the mean of the AGA group, which was assigned an arbitrary value of 1 .

\section{Statistical analyses}

Data were analyzed using the SigmaStat Software, v3.1 (San Jose, CA, USA). Demographic data were analyzed using the unpaired Student's $t$-test to compare continuous variables and the Fisher's exact test to compare contingency tables. Quantitative data expressed as relative values were used for analysis using the two-way ANOVA followed by the HolmSidak all pairwise multiple comparisons post hoc analysis. The quantitative datasets passed the normality and equal variance tests. Spearman's rank correlation test was used to determine significant correlations between the variables. Significance was taken as $P<0.05$.

\section{Results}

\section{MCT8 immunolocalization within human fetal and adult cerebral cortex}

The developing human fetal cerebral cortex in midgestation is formed by several layers; from superficial to deep, they are the marginal zone, cortical plate, subplate, intermediate zone, subventricular zone, and ventricular zone (lying adjacent to the ventricle; Bystron et al. 2008). At 19 weeks of gestation, sections of the parietal and occipital cortex obtained from AGA fetuses demonstrated MCT8 immunostaining in all the layers. Immunostaining was found within the marginal zone, in cortical plate neurons, a proportion of cells in the subplate zone, in hippocampal neurons, epithelial cells of the choroid plexus and ependyma, and in numerous cells in the ventricular and subventricular zones (Fig. 1A, B, C, D and H). A similar distribution of MCT8 immunostaining was observed in sections obtained from AGA fetuses at 26-37 weeks of gestation. However, with advancing gestation and maturity of the cortex, there were fewer cells in the ventricular and subventricular zones and, hence, correspondingly less MCT8 staining in these layers (Fig. 1D, E and F). Most microvessels throughout the areas studied were MCT8 positive (Fig. 1G). The absorption of the antibody with the blocking peptide effectively abolished MCT8 staining, confirming the specificity of staining (Fig. 1A, B and C).

MCT8 immunostaining corresponded with the welldescribed pattern of neuronal cell distribution within the cerebral cortex, with the greatest staining being observed in the cortical plate, which is dense with neurons. Neuronal localization of MCT8 was also supported by our findings that immunostaining for GFAP and CD68, indicating glia and microglia respectively, in adjacent sections revealed an entirely different pattern of distribution in all the layers of the cortex (Fig. 1J and K) compared with immunostaining for MCT8. Specifically, there was no GFAP (Fig. 1L) or CD68 immunostaining in the cortical plate. In addition, the morphology of cells stained with each antibody was clearly different. Neurons were identified by a round dense nucleus and abundant cytoplasm with dendritic branches, many of which were immunostained for MCT8, in contrast to astrocytes, which had a large irregular nucleus with clear nucleoplasm containing a vesicular chromatin pattern and very small or absent nucleoli showing no MCT8 immunostaining (Fig. 1H and I). Within the subplate only a selected population of neurons were MCT8 positive at every gestational age examined. In the adult occipital cortex, microvessels were immunostained for MCT8, but proportionally fewer neurons were immunostained for MCT8 compared with those in the fetal cortex (data not shown).

\section{Comparison of MCT8 immunostaining in the occipital cortex of AGA and IUGR fetuses}

There were no significant differences between the IUGR and AGA cohorts in terms of gestational age and fetal sex (Table 1). Compared with the AGA group, the IUGR group had significantly lower raw birth weights $(P<0.05$; with all the customized birth weight percentiles being under the third percentile), but the raw brain weights were not significantly different between the two groups, with brain weights being well preserved for gestation even in the IUGR cohort (1.08 relative to the expected mean). However, the relative brain weights (ratio to the expected mean for gestation) in the IUGR group were still lower compared with those in the AGA group $(P<0.05)$. The brain:liver weight ratios in the IUGR group were significantly higher compared with those in the AGA group $(P<0.01)$. Atrophy of the thymus secondary to chronic stress in IUGR (Cox \&

Published by Bioscientifica Ltd 

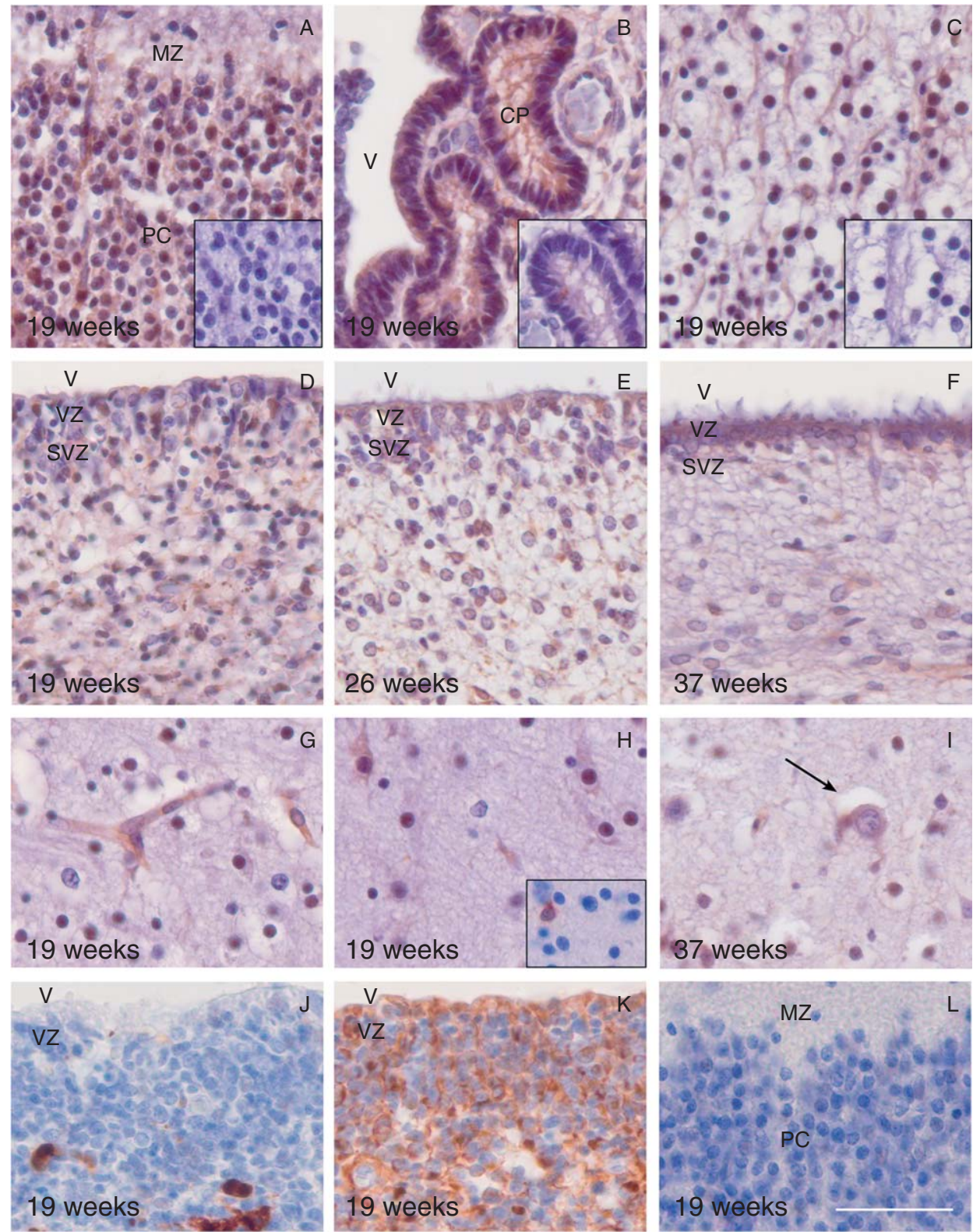

\section{Figure 1}

MCT8 immunohistochemistry of cerebral cortex sections obtained from structurally normal fetuses with unexplained intrauterine death. Corresponding negative controls (antibody absorption by the blocking peptide) of adjacent sections are shown in panel inserts in the bottom right corner for (A, B and C). At 19 weeks, MCT8 was located in the cortical plate within the parietal cortex $(\mathrm{PC})$ with less staining in the marginal zone (MZ) (A), in the choroid plexus (CP) (B), and the hippocampus (C). МCT8 immunostaining was also observed in the ependymal cells lining the ventricle (V) and in numerous cells within the ventricular zone (VZ) and subventricular zone (SVZ) at 19 weeks (D), 26 weeks (E), and 37 weeks (F). MCT8

Marton 2009) was also evident by the significantly reduced raw thymus weights $(P<0.01)$ and thymus weights relative to the expected mean for gestation $(P<0.001)$. All these indicate that the IUGR cohort comprised cases at the severe immunostaining was observed in the wall of a microvessel in the subplate at 19 weeks $(G)$ and in neurons in the intermediate zone at 19 weeks $(H)$ and 37 weeks ( $\mathrm{l}$; arrow points to a neuron). An adjacent section stained with GFAP is shown in a panel insert in the bottom right corner for $(\mathrm{H})$ showing differences in the morphology of immunostained cells. At 19 weeks, CD68 immunostaining for microglia (J) and GFAP immunostaining for glia $(K)$ in the ventricular and subventricular zones showed a different pattern of staining compared to that of MCT8. There was also a lack of GFAP staining in the cortical plate and marginal zone of the parietal cortex at 19 weeks (L). Magnification bar $=50 \mu \mathrm{m}$.

end of the spectrum. Most of the IUGR cases demonstrated features of chronic uteroplacental failure on placental examination (Table 1; ReCoDe C4 and C5), which were absent in the AGA cohort.

Published by Bioscientifica Ltd 


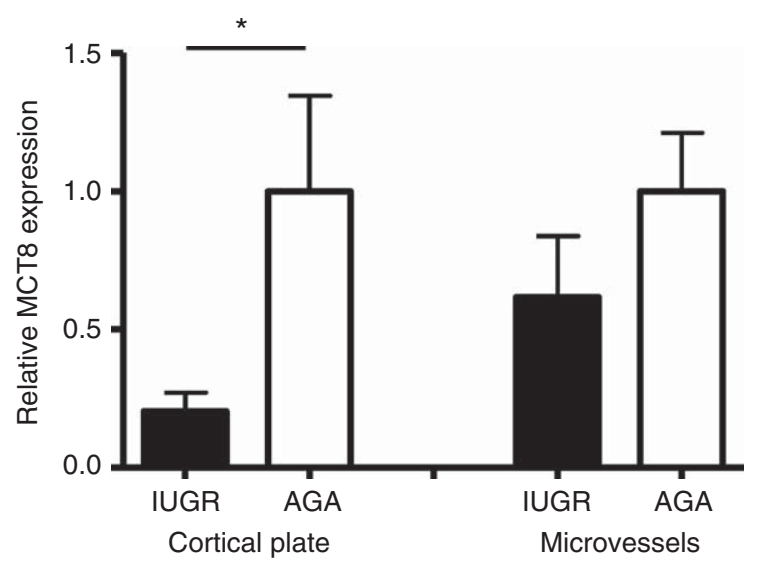

Figure 2

Quantification of MCT8 immunostaining in the occipital cerebral cortex of intrauterine growth-restricted (IUGR) fetuses ( $n=7$; black bars) compared with that in the occipital cerebral cortex of appropriately grown for gestational age (AGA) fetuses ( $n=5$; white bars). The percentage area of cortical plate and the proportion of microvessels in the subplate immunostained for MCT8 are expressed relative to the mean of the AGA group, which was given an arbitrary value of 1 . Columns and error bars represent the mean and S.E.M. Statistically significant difference, ${ }^{*} P<0.05$.

The overall two-way ANOVA, which analyzed the entire dataset, indicated significantly reduced MCT8 expression in the occipital cortex of IUGR fetuses compared with that in AGA fetuses $(P<0.05)$. However, post hoc tests indicated that the difference was significant only for cortical plate immunostaining $(P<0.05$; Fig. 2$)$.

The mean percentage area of cortical plate immunostaining after correction for relative cell number was $4.7 \pm 1.5 \%$ (mean \pm s.E.M.; $0.2 \pm 0.07$ relative to $A G A$ ) in the IUGR group compared with $23.3 \pm 8.1 \%$ ( $1 \pm 0.3$ relative to AGA) in the AGA group $(P<0.05)$, which represents approximately a fivefold decrease in MCT8 expression with IUGR (Fig. 2). The cellularity within the cortical plate was not significantly different between the two cohorts (IUGR, $1.3 \pm 0.1$ and AGA, $1 \pm 0.1$ ). General observations of cortical plate images indicated that the decrease in MCT8 staining was confined to morphologically defined neuronal cells, while microvessels seemed to be spared (Fig. 3).

Post hoc tests revealed no statistically significant difference in the proportion of microvessels stained for МСT8 in the IUGR samples $(27.9 \pm 10.0 \% ; 0.6 \pm 0.2$ relative to AGA) compared with that in the AGA samples $(45.2 \pm 9.6 \% ; 1 \pm 0.2$ relative to AGA; Fig. 2$)$.

However, there was a significant positive correlation between the area of cortical plate MCT8 immunostaining and the proportion of microvessels stained in the subplate (correlation coefficient $=0.71, r^{2}=0.27 ; P<0.01$ ) when all
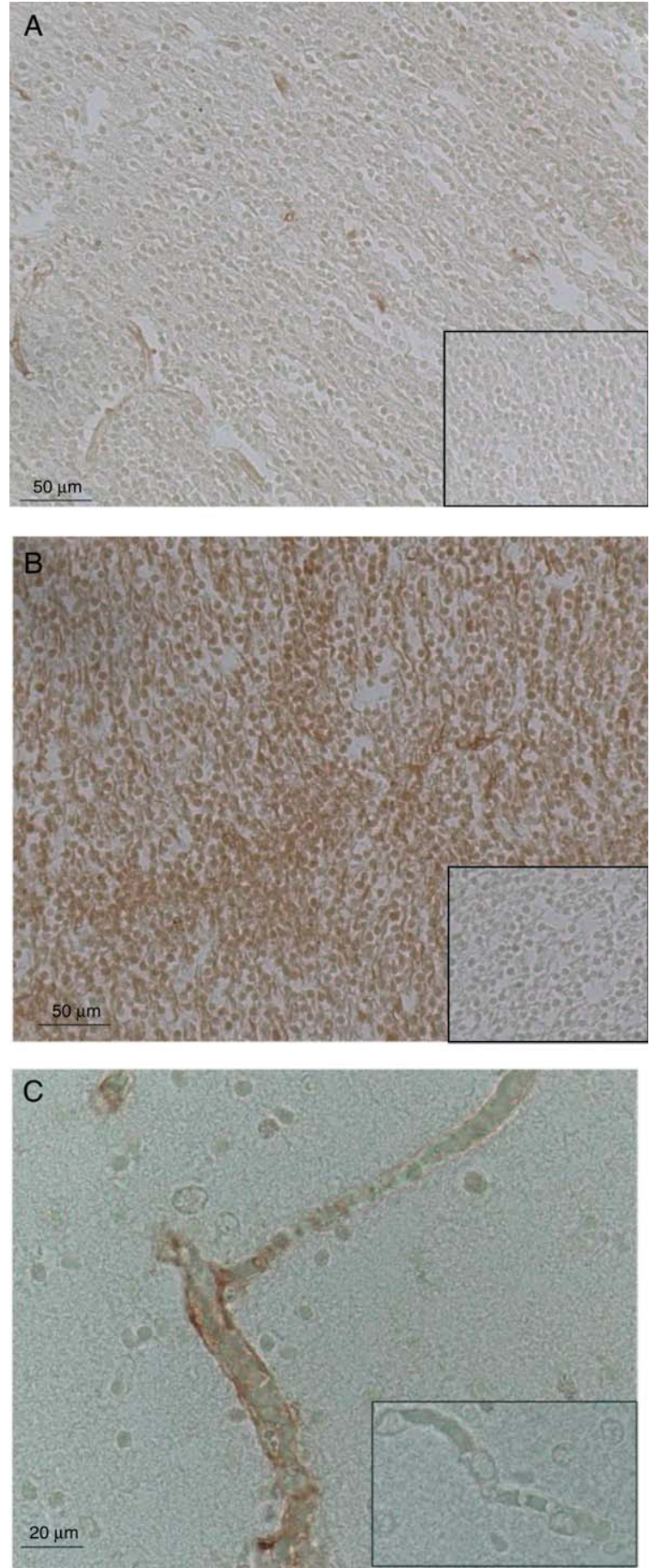

\section{Figure 3}

Representative sections showing MCT8 immunostaining of the cortical plate of an intrauterine growth-restricted (IUGR) fetus (A) and an appropriately grown for gestational age (AGA) fetus (B) within the occipital cerebral cortex. Corresponding negative controls (no primary antibody) of adjacent sections are shown in a panel insert in the bottom right corner. An example of a positively MCT8-immunostained microvessel in the subplate (C) compared with a negative one (shown in a panel insert in the bottom right corner) from the same section immunostained for MCT8 is shown.

Published by Bioscientifica Ltd 

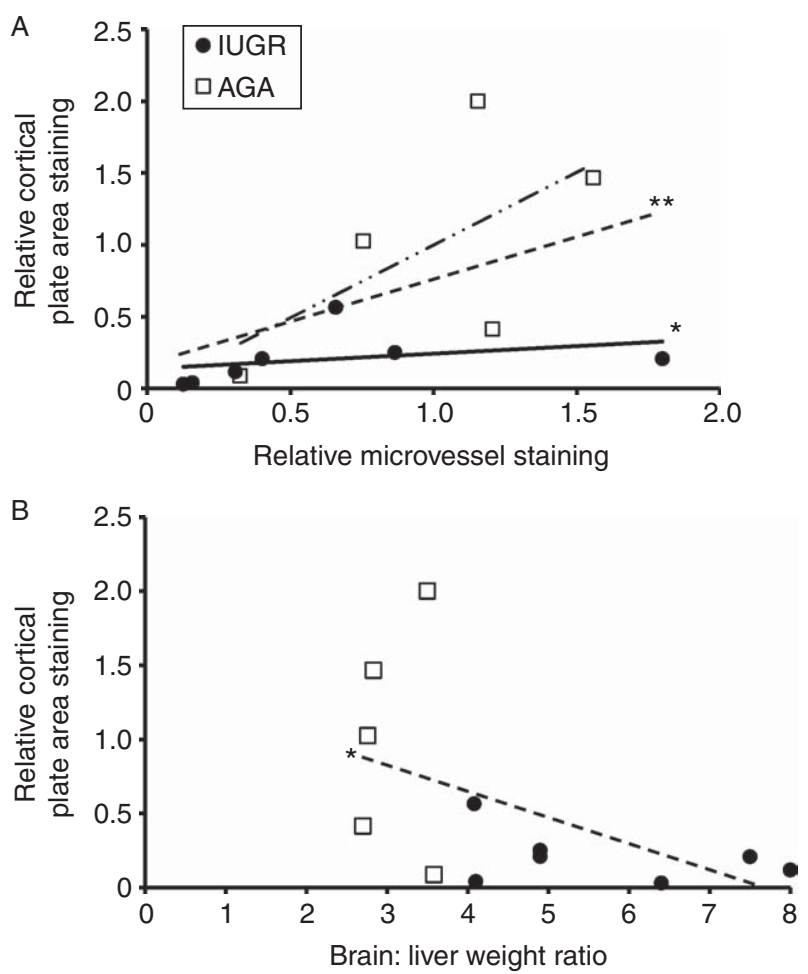

\section{Figure 4}

(A) Correlation between the relative cortical plate area immunostained for MCT8 and the relative proportion of microvessels immunostained for MCT8 in IUGR (black dots) and AGA (white squares) fetuses. A significant positive correlation was observed when all the samples were considered together (dashed line) and when only the IUGR samples were considered on their own (straight line), but there was no significant correlation when only among the AGA samples were considered on their own (dashed and dotted line). (B) Correlation between the relative cortical plate area immunostained for MCT8 and the brain:liver weight ratio. A negative correlation was observed when all the samples were considered together. Statistically significant differences are $* * P<0.01, * P<0.05$.

the samples were analyzed together. The positive correlation remained significant within the IUGR group (correlation coefficient $=0.75, r^{2}=0.12 ; P<0.05$; Fig. 4A), but there was no significant correlation within the AGA cohort on its own.

When all the samples were analyzed together, a negative correlation was also observed between the area of cortical plate MCT8 immunostaining and brain:liver weight ratios (correlation coefficient $=-0.64, r^{2}=0.28$; $P<0.05$; Fig. 4B). There was no correlation between MCT8 immunostaining in the cortical plate or microvessels with either gestational age or fetal sex.

\section{Discussion}

Changes in $\mathrm{TH}$ transporter expression have never been described in the growth-restricted state. This study is the first to demonstrate significantly reduced cortical MCT8 expression within the developing CNS of human fetuses stillborn with severe IUGR. Our results suggest that altered TH transporter activity in cerebral neurons could be a contributory factor to the pathophysiology of neurodevelopmental impairment associated with IUGR.

The strength of this study is the use of human fetal tissue samples, thus eliminating species differences, particularly relevant, as Mct8-knockout mice lack the neurological phenotype observed in humans with MCT8 mutations. However, a limitation is the restriction of the availability of human fetal tissue samples of adequate quality for investigation, hence, the small number of samples in this study.

The localization of MCT8 in developing neurons across the different cortical layers, microvessels, and choroid plexus reported herein is generally consistent with the findings of previously published studies of human fetuses (Roberts et al. 2008, Wirth et al. 2009) and supports its role in the uptake of TH into the brain parenchyma from the blood and cerebrospinal fluid, as well as into neurons, from early fetal development.

Neurogenesis takes place in the ventricular and subventricular zones with much being completed by 28 weeks of gestation (Bystron et al. 2008). MCT8 staining in these neuroprecursor-rich areas at 19-26 weeks suggests its involvement in the regulation of neurogenesis. Indeed, we have previously demonstrated that MCT8 represses the proliferation of the human neuronal precursor cell NT2 in a $\mathrm{T}_{3}$-independent manner (James et al. 2009); however, MCT8 had no effect on NT2 neurodifferentiation in vitro (Chan et al. 2011). Post-mitotic neurons migrate away from the proliferative zones and, by $24-28$ weeks of gestation, most cortical neurons have settled to form the cortical plate, an area comprising predominantly neuronal cells (Bystron et al. 2008).

During normal human fetal cortical development, over $70 \%$ of neurons undergo programmed cell death after 32 weeks of gestation (Rabinowicz et al. 1996). Magnetic resonance imaging (MRI) assessments of IUGR premature infants at 33-34 weeks have shown reduced cerebral cortical gray matter volume (Tolsa et al. 2004), which could be due to reduced cell numbers in the cortical plate (Samuelsen et al. 2003). Similar to that observed in our IUGR cohort at 24-28 weeks, that study (Samuelsen et al. 2003), however, found no significant differences in cortical cell numbers compared with AGA samples before 27 weeks.

MCT8 promotes cell death in non-proliferative cytotrophoblast cells from human placenta 
independently of $\mathrm{T}_{3}$ (Vasilopoulou et al. 2013). It remains speculative whether MCT8 could also have a similar effect on neuronal apoptosis. If so, the downregulation of MCT8 expression in IUGR neurons could be a protective mechanism to limit neuronal apoptosis at the expense of TH transport, of which the latter could be partially compensated for by other $\mathrm{TH}$ transporters expressed by neurons, as we and others have previously described in the human fetal cerebral cortex (Wirth et al. 2009, Chan et al. 2011).

Whether the reduction in cortical cell number in the third trimester is due to reduced neurogenesis, reduced neuronal migration, or increased cell death in IUGR is not known. In rats, abnormal neuronal migration in the fetal CNS in both IUGR (Sasaki et al. 2000) and TH deficiency (Auso et al. 2004) has been reported. Maternal TH deficiency in rats has also been reported to lead to impaired neurogenesis and diminished neocortical neuronal numbers (Mohan et al. 2012). The extent to which these altered cellular processes in IUGR, as well as possibly altered synaptogenesis and dendritic branching, are mediated by diminished $\mathrm{TH}$ action secondary to reductions in circulating TH concentrations, MCT8 transport, and TR expression remains the subject of investigation. Other factors such as cerebral hypoxia and prematurity are also likely to contribute to this neuropathology. Whatever the etiologies, alterations in brain neural networks assessed by MRI in IUGR infants have been found to be associated with later neurodevelopmental outcomes (Batalle et al. 2012).

Current understanding of the physiological regulation of MCT8 expression is poor. TH status has been shown to influence MCT8 expression in some tissues (Capelo et al. 2009) but not in others (Mebis et al. 2009). In IUGR, MCT8 expression in the human placenta is upregulated (Vasilopoulou et al. 2010) in contrast to that in the fetal cerebral cortex. These tissue-specific effects argue against a general alteration in MCT8 activity being part of the etiology of IUGR, but rather suggest that altered cerebral MCT8 expression is a local adaptive response to the growth-restricted state that is associated with chronic distress, which is supported by our finding that the greater the growth restriction, the lower the MCT8 expression. This is in contrast to the AGA fetuses that presumably suffered from an acute event just before death. The positive correlation between MCT8 expression in the cortical plate and that in microvessels suggests that there may be some common mechanisms regulating МСT8 expression in the CNS.
Future studies should investigate whether there are compensatory alterations in the expression of other $\mathrm{TH}$ transporters in neurons and microvessels. Studies could also extend to other regions of the CNS and at different gestational ages to obtain a more comprehensive picture of the effects of IUGR on TH transport and how this could correlate with observed neurological impairments in IUGR survivors.

In conclusion, our results showing perturbed patterns of cortical MCT8 expression support the hypothesis that a reduction in MCT8 expression in the IUGR fetal CNS could be a contributory factor implicated in the long-term neurodevelopmental impairments associated with this condition.

\section{Declaration of interest}

The authors declare that there is no conflict of interest that could be perceived as prejudicing the impartiality of the research reported.

\section{Funding}

This work was supported by the Health Foundation (Clinician Scientist Fellowship awarded to S Y C; 6462/4335), Action Medical Research (SP4142 to J A F, S Y C, M D K, C J M, and A L), Medical Research Council UK (G0501548 to S Y C, M D K, J A F, and C J M), and the University of Birmingham.

\section{Acknowledgements \\ The authors thank Aga Hussain for assistance with the immunohistochemistry.}

\section{References}

Auso E, Lavado-Autric R, Cuevas E, del Rey FE, Morreale De EG \& Berbel P 2004 A moderate and transient deficiency of maternal thyroid function at the beginning of fetal neocorticogenesis alters neuronal migration. Endocrinology 145 4037-4047. (doi:10.1210/en.2004-0274)

Batalle D, Eixarch E, Figueras F, Munoz-Moreno E, Bargallo N, Illa M, Acosta-Rojas R, Amat-Roldan I \& Gratacos E 2012 Altered small-world topology of structural brain networks in infants with intrauterine growth restriction and its association with later neurodevelopmental outcome. NeuroImage 60 1352-1366. (doi:10.1016/j.neuroimage. 2012.01.059)

Bystron I, Blakemore C \& Rakic P 2008 Development of the human cerebral cortex: Boulder Committee revisited. Nature Reviews. Neuroscience 9 110-122. (doi:10.1038/nrn2252)

Capelo LP, Beber EH, Fonseca TL \& Gouveia CH 2009 The monocarboxylate transporter 8 and L-type amino acid transporters 1 and 2 are expressed in mouse skeletons and in osteoblastic MC3T3-E1 cells. Thyroid 19 171-180. (doi:10.1089/thy.2008.0120)

Ceballos A, Belinchon MM, Sanchez-Mendoza E, Grijota-Martinez C, Dumitrescu AM, Refetoff S, Morte B \& Bernal J 2009 Importance of monocarboxylate transporter 8 for the blood-brain barrier-dependent availability of 3,5,3'-triiodo-L-thyronine. Endocrinology 150 2491-2496. (doi:10.1210/en.2008-1616)

Published by Bioscientifica Ltd 
Chan S, Kachilele S, McCabe C, Tannahill L, Boelaert K, Gittoes N, Visser T, Franklyn J \& Kilby M 2002 Early expression of thyroid hormone deiodinases and receptors in human fetal cerebral cortex. Brain Research. Developmental Brain Research 138 109-116. (doi:10.1016/ S0165-3806(02)00459-5)

Chan SY, Andrews MH, Lingas R, McCabe CJ, Franklyn JA, Kilby MD \& Matthews SG 2005 Maternal nutrient deprivation induces sex-specific changes in thyroid hormone receptor and deiodinase expression in the fetal guinea pig brain. Journal of Physiology 566 467-480. (doi:10.1113/ jphysiol.2005.084673)

Chan SY, Martin-Santos A, Loubiere LS, Gonzalez AM, Stieger B, Logan A, McCabe CJ, Franklyn JA \& Kilby MD 2011 The expression of thyroid hormone transporters in the human fetal cerebral cortex during early development and in N-Tera-2 neurodifferentiation. Journal of Physiology 589 2827-2845. (doi:10.1113/jphysiol.2011.207290)

Cox P \& Marton T 2009 Pathological assessment of intrauterine growth restriction. Best Practice \& Research. Clinical Obstetrics \& Gynaecology 23 751-764. (doi:10.1016/j.bpobgyn.2009.06.006)

Dowdeswell HJ, Slater AM, Broomhall J \& Tripp J 1995 Visual deficits in children born at less than 32 weeks' gestation with and without major ocular pathology and cerebral damage. British Journal of Ophthalmology 79 447-452. (doi:10.1136/bjo.79.5.447)

Dumitrescu AM, Liao XH, Best TB, Brockmann K \& Refetoff S 2004 A novel syndrome combining thyroid and neurological abnormalities is associated with mutations in a monocarboxylate transporter gene. American Journal of Human Genetics 74 168-175. (doi:10.1086/380999)

Ferreira T \& Rasband W 2012 Quantifying stained liver tissue. In ImageJ User Guide. Research Services Branch, National Institute of Health, Bethesda, MD, USA. (Available at: http://rsbweb.nih.gov/ij/docs/examples/ stained-sections/index.html).

Friesema EC, Ganguly S, Abdalla A, Manning Fox JE, Halestrap AP \& Visser TJ 2003 Identification of monocarboxylate transporter 8 as a specific thyroid hormone transporter. Journal of Biological Chemistry 278 40128-40135. (doi:10.1074/jbc.M300909200)

Friesema EC, Grueters A, Biebermann H, Krude H, von Moers A, Reeser M, Barrett TG, Mancilla EE, Svensson J, Kester MH et al. 2004 Association between mutations in a thyroid hormone transporter and severe X-linked psychomotor retardation. Lancet 364 1435-1437. (doi:10. 1016/S0140-6736(04)17226-7)

Garcia-Silva S, Perez-Juste G \& Aranda A 2002 Cell cycle control by the thyroid hormone in neuroblastoma cells. Toxicology 181-182 179-182. (doi:10.1016/S0300-483X(02)00277-9)

Gardosi J, Chang A, Kalyan B, Sahota D \& Symonds EM 1992 Customized antenatal growth charts. Lancet 339 283-287. (doi:10.1016/01406736(92)91342-6)

Gardosi J, Kady SM, McGeown P, Francis A \& Tonks A 2005 Classification of stillbirth by relevant condition at death (ReCoDe): population based cohort study. BMJ331 1113-1117. (doi:10.1136/bmj.38629.587639.7C)

Gilbert ME \& Paczkowski C 2003 Propylthiouracil (PTU)-induced hypothyroidism in the developing rat impairs synaptic transmission and plasticity in the dentate gyrus of the adult hippocampus. Developmental Brain Research 145 19-29. (doi:10.1016/S01653806(03)00191-3)

Heuer H \& Mason CA 2003 Thyroid hormone induces cerebellar Purkinje cell dendritic development via the thyroid hormone receptor alpha1. Journal of Neuroscience 23 10604-10612.

James SR, Franklyn JA, Reaves BJ, Smith VE, Chan SY, Barrett TG, Kilby MD \& McCabe CJ 2009 Monocarboxylate transporter 8 in neuronal cell growth. Endocrinology 150 1961-1969. (doi:10.1210/en.2008-1031)

Jones SA, Jolson DM, Cuta KK, Mariash CN \& Anderson GW 2003 Triiodothyronine is a survival factor for developing oligodendrocytes. Molecular and Cellular Endocrinology 199 49-60. (doi:10.1016/ S0303-7207(02)00296-4)

Kady M \& Gardosi J 2004 Perinatal mortality and fetal growth restriction. Best Practice \& Research. Clinical Obstetrics \& Gynaecology 18 397-410. (doi:10.1016/j.bpobgyn.2004.02.009)
Kilby MD, Verhaeg J, Gittoes N, Somerset DA, Clark PM \& Franklyn JA 1998 Circulating thyroid hormone concentrations and placental thyroid hormone receptor expression in normal human pregnancy and pregnancy complicated by intrauterine growth restriction (IUGR). Journal of Clinical Endocrinology and Metabolism 83 2964-2971. (doi:10.1210/jc.83.8.2964)

Kilby MD, Gittoes N, McCabe C, Verhaeg J \& Franklyn JA 2000 Expression of thyroid receptor isoforms in the human fetal central nervous system and the effects of intrauterine growth restriction. Clinical Endocrinology 53 469-477. (doi:10.1046/j.1365-2265.2000.01074.x)

Kok JH, den Ouden AL, Verloove-Vanhorick SP \& Brand R 1998 Outcome of very preterm small for gestational age infants: the first nine years of life. British Journal of Obstetrics and Gynaecology 105 162-168. (doi:10.1111/ j.1471-0528.1998.tb10046.x)

Lavado-Autric R, Auso E, Garcia-Velasco JV, Arufe MD, del Rey FE, Berbel P \& de Escobar GM 2003 Early maternal hypothyroxinemia alters histogenesis and cerebral cortex cytoarchitecture of the progeny. Journal of Clinical Investigation 111 1073-1082. (doi:10.1172/JCI16262)

Leitner Y, Fattal-Valevski A, Geva R, Eshel R, Toledano-Alhadef H, Rotstein M, Bassan H, Radianu B, Bitchonsky O, Jaffa AJ et al. 2007

Neurodevelopmental outcome of children with intrauterine growth retardation: a longitudinal, 10-year prospective study. Journal of Child Neurology 22 580-587. (doi:10.1177/0883073807302605)

Mebis L, Debaveye Y, Ellger B, Derde S, Ververs EJ, Langouche L, Darras VM, Fliers E, Visser TJ \& Van den BG 2009 Changes in the central component of the hypothalamus-pituitary-thyroid axis in a rabbit model of prolonged critical illness. Critical Care 13 R147. (doi:10.1186/cc8043)

Mohan V, Sinha RA, Pathak A, Rastogi L, Kumar P, Pal A \& Godbole MM 2012 Maternal thyroid hormone deficiency affects the fetal neocorticogenesis by reducing the proliferating pool, rate of neurogenesis and indirect neurogenesis. Experimental Neurology 237 477-488. (doi:10.1016/j.expneurol.2012.07.019)

Rabinowicz T, de Courten-Myers GM, Petetot JM, Xi G \& de los Reyes E 1996 Human cortex development: estimates of neuronal numbers indicate major loss late during gestation. Journal of Neuropathology and Experimental Neurology 55 320-328. (doi:10.1097/00005072199603000-00007)

Roberts LM, Woodford K, Zhou M, Black DS, Haggerty JE, Tate EH, Grindstaff KK, Mengesha W, Raman C \& Zerangue N 2008 Expression of the thyroid hormone transporters monocarboxylate transporter- 8 (SLC16A2) and organic ion transporter-14 (SLCO1C1) at the blood-brain barrier. Endocrinology 149 6251-6261. (doi:10.1210/ en.2008-0378)

Samuelsen GB, Larsen KB, Bogdanovic N, Laursen H, Graem N, Larsen JF \& Pakkenberg B 2003 The changing number of cells in the human fetal forebrain and its subdivisions: a stereological analysis. Cerebral Cortex 13 115-122. (doi:10.1093/cercor/13.2.115)

Sasaki J, Fukami E, Mimura S, Hayakawa M, Kitoh J \& Watanabe K 2000 Abnormal cerebral neuronal migration in a rat model of intrauterine growth retardation induced by synthetic thromboxane A(2). Early Human Development 58 91-99. (doi:10.1016/S03783782(00)00069-4)

Schwartz CE, May MM, Carpenter NJ, Rogers RC, Martin J, Bialer MG, Ward J, Sanabria J, Marsa S, Lewis JA et al. 2005 Allan-Herndon-Dudley syndrome and the monocarboxylate transporter 8 (MCT8) gene. American Journal of Human Genetics 77 41-53. (doi:10.1086/431313)

Tolsa CB, Zimine S, Warfield SK, Freschi M, Sancho RA, Lazeyras F, Hanquinet S, Pfizenmaier M \& Huppi PS 2004 Early alteration of structural and functional brain development in premature infants born with intrauterine growth restriction. Pediatric Research 56 132-138. (doi:10.1203/01.PDR.0000128983.54614.7E)

Trajkovic M, Visser TJ, Mittag J, Horn S, Lukas J, Darras VM, Raivich G, Bauer K \& Heuer H 2007 Abnormal thyroid hormone metabolism in mice lacking the monocarboxylate transporter 8. Journal of Clinical Investigation 117 627-635. (doi:10.1172/JCI28253) 
Vasilopoulou E, Loubiere LS, Martin-Santos A, McCabe CJ, Franklyn JA, Kilby MD \& Chan SY 2010 Differential triiodothyronine responsiveness and transport by human cytotrophoblasts from normal and growth-restricted pregnancies. Journal of Clinical Endocrinology and Metabolism 95 4762-4770. (doi:10.1210/jc.2010-0354)

Vasilopoulou E, Loubiere LS, Heuer H, Trajkovic-Arsic M, Darras VM, Visser TJ, Lash GE, Whitley GS, McCabe CJ, Franklyn JA et al. 2013 Monocarboxylate transporter 8 modulates the viability and invasive capacity of human placental cells and fetoplacental growth in mice. PLOS ONE 8 e65402. (doi:10.1371/journal.pone.0065402)
Wirth EK, Roth S, Blechschmidt C, Holter SM, Becker L, Racz I, Zimmer A, Klopstock T, Gailus-Durner V, Fuchs H et al. 2009 Neuronal 3',3,5-triiodothyronine $\left(T_{3}\right)$ uptake and behavioral phenotype of mice deficient in Mct8, the neuronal $\mathrm{T}_{3}$ transporter mutated in Allan-Herndon-Dudley syndrome. Journal of Neuroscience $\mathbf{2 9}$ 9439-9449. (doi:10.1523/JNEUROSCI.6055-08.2009)

Zoeller RT \& Rovet J 2004 Timing of thyroid hormone action in the developing brain: clinical observations and experimental findings. Journal of Neuroendocrinology 16 809-818. (doi:10.1111/j.1365-2826. 2004.01243.x)

Received in final form 31 October 2013

Accepted 7 November 2013

Accepted Preprint published online 7 November 2013
(C) 2014 The authors Printed in Great Britain
Published by Bioscientifica Ltd 